one mainly responsible for thermotransport of $\mathrm{Au}$ in Li. Further experimental and theoretical work in this field should be illuminating.

This work has been supported by the Swedish Council for Applied Research and by the Adlerbertska forskningsfonden.
I gratefully acknowledge the encouragement of Docent A. LODDING, with whom I have had many stimulating discussions. Thanks are also due to Drs. I. Torrens and M. GERL (Saclay) and to Dr. R. FEDER (Yorktown Heights) for showing their recent results prior to publication. Ing. H. OLsson has been of considerable assistance in several stages of the experiments.

\section{Desorientierung von optisch ausgerichteten Na-Atomen durch Edelgas-Stöße}

\section{H. SOBOLL}

I. Physikalisches Institut der Universität Gießen

(Z. Naturforsch. 24 a, 2023-2024 [1969] ; eingeg. am 23. Oktober 1969)

Cross sections for noble-gas-induced sodium spin disorientation have been measured and calculated by applying the spin-orbit and spin-exchange-model of R. Herman.

Index headings: Atomic physics, optical pumping.

Die Relaxation der Spinpolarisation $\left\langle S_{z}\right\rangle$ durch Edelgasstöße ist für $\mathrm{S}_{1 / 2}$-Grundzustände wesentlich schwächer als für $P_{j}$-Zustände $(j \neq 0)$. Während die Desorientierungsquerschnitte der P-Zustände von der Größenorddung des gaskinetischen Querschnittes $\left(\sim 10^{-16} \mathrm{~cm}^{2}\right)$ sind, liegen die entsprechenden Werte für $\mathrm{S}_{1 / 2}$-Grundzustände der Alkaliatome zwischen $10^{-20} \mathrm{~cm}^{2}$ und $10^{-26} \mathrm{~cm}^{2}$. Zur Erklärung dieser kleinen Wirkungsquerschnitte hat HERMAN zwei verschiedene Wechselwirkungen vorgeschlagen: Einmal den Spinaustausch zwischen dem Valenzelektron und dem Edelgas-Kernspin $^{1}$ und zum anderen eine Kopplung des Valenzelektronenspins mit einem stoßinduzierten Bahnmoment ${ }^{2}$. Im folgenden werden analog der Theorie von Herman Desorientierungsquerschnitte für Na-EdelgasStöße berechnet und mit experimentellen Werten verglichen.

Die Spin-Bahn-Kopplung liefert in erster störungstheoretischer Näherung einen vernachlässigbaren Beitrag zur Relaxation. Der Wechselwirkungsterm zweiter Ordnung kann geschrieben werden

$$
H_{\text {eff }}=\gamma(R) \boldsymbol{K} \cdot \boldsymbol{S}, \boldsymbol{K} / \hbar=\boldsymbol{r} \times \boldsymbol{p},
$$

wobei $\boldsymbol{S}$ der Spin des Valenzelektrons und $\boldsymbol{K}$ der Gesamtbahndrehimpuls des Stoßpaares bedeutet. Zur expliziten Berechnung der vom Kernabstand $R$ abhängigen Faktoren $\gamma(R)$ betrachtet Herman sowohl Deformationen wie auch Überlappungen der beiden Stoßpartner. Obwohl bei den leichten Alkaliatomen Überlappungseffekte sicher eine größere Rolle spielen als bei $R b$ und $C s$, werden sie hier vernachlässigt, da zu ihrer Berechnung genaue Wellenfunktionen notwendig sind.

Sonderdruckanforderungen an H. SовоLl, I. Physikalisches Institut der Universität Gießen, D-6300 Gießen, Leihgesterner Weg 104.

1 R. M. Herman, Phys. Rev. 137, A 1062 [1965].

2 R. M. Herman, Phys. Rev. 136, A 1576 [1964].
Eine Abschätzung der Deformationen auf Grund weitreichender Coulomb-Kräfte führt zu

$$
\gamma(R) \sim-(2 / 9)\left[\Delta E_{\text {disp }}(R) \cdot E_{\mathrm{res}}\left({ }^{2} \mathrm{p}\right)\right](E+I)^{-2}
$$

mit der Dispersionsenergie $\Delta E_{\text {disp }}=-C_{6} / R^{6}$, der Feinstrukturaufspaltung $E_{\text {res }}\left({ }^{2} p\right)$, der Edelgasionisationsenergie $I$, und der Alkali-Resonanzenergie $E$. Für NaHe-Stöße errechnet sich dieser Beitrag zu

$$
\gamma\left(b_{0}\right)=7,5 \cdot 10^{-9} \text {. }
$$

Der kinetische Radius $b_{0}$ wurde durch Mittelung der beiden Lennard-Jones-Radien ${ }^{3}$ erhalten, während die van der Waals-Konstanten $C_{6}$ und $C_{8}$ der Literatur ${ }^{4}$ entnommen wurden.

Zur Berechnung der abstoßenden Nahkräfte wird zunächst die Wechselwirkungsenergie $E^{\prime}$ ohne Deformation für das Na-He-System im Abstand $b_{0}$ ermittelt:

$$
E^{\prime}=+0,012 \text { at. E. }
$$

Dabei wurde als He-Wellenfunktion die 1s-Wasserstofffunktion mit der effektiven Kernladung $Z=27 / 16$ und für $\mathrm{Na}$ eine $3 \mathrm{~s}$-Wasserstoffunktion mit $Z=1,846$ benutzt. Dies ergibt einen Wert für die Stärke der kurz-

\begin{tabular}{|c|c|c|c|c|c|}
\hline & & $\sigma_{\mathrm{SB}}$ & $\sigma_{\mathrm{SS}}$ & $\sigma_{\text {theor. }}$ & $\sigma_{\exp }$ \\
\hline $\mathrm{He}$ & 36 & $\cdot 10^{-26}$ & $3,8 \cdot 10^{-23}\left(\mathrm{He}^{3}\right)$ & $36 \cdot 10^{-26}$ & $\sim 10 \cdot 10^{-26}$ \\
\hline $\mathrm{Ne}$ & 3 & $4 \cdot 10^{-24}$ & $4,5 \cdot 10^{-22}\left(\mathrm{Ne}^{21}\right)$ & $4,5 \cdot 10^{-24}$ & $(3,6 \pm 1,1) \cdot 10^{-24}$ \\
\hline Ar & 13 & $\cdot 10^{-23}$ & - & $13 \cdot 10^{-23}$ & $(9,6 \pm 4,3) \cdot 10^{-23}$ \\
\hline $\mathrm{Kr}$ & 4 & $\cdot 10^{-22}$ & $1,2 \cdot 10^{-20}\left(\mathrm{Kr}^{83}\right)$ & $1,7 \cdot 10^{-21}$ & $(1,5 \pm 0,3) \cdot 10^{-21}$ \\
\hline $\mathrm{Xe}$ & 9 & $\cdot 10^{-22}$ & $5,9 \cdot 10^{-20}\left(\mathrm{Xe}^{129}\right)$ & $2,9 \cdot 10^{-20}$ & $(6,6 \pm 1,1) \cdot 10^{-20}$ \\
\hline
\end{tabular}
reichweitigen Wechselwirkung bei $\mathrm{Na}-\mathrm{He}$ von $\gamma\left(b_{0}\right)$ $=2,6 \cdot 10^{-5}$ und übertrifft also den weitreichenden Beitrag um vier Größenordnungen.

Tab. 1. Wirkungsquerschnitte in $\mathrm{cm}^{2}$.

Mit der Streuphase $\Phi$ kann der Desorientierungsquerschnitt $\sigma$ SB nach der Stoßparameter-Methode abgeschätzt werden :

$$
\sigma_{\mathrm{SB}}=\frac{4 \pi}{3} \int_{0}^{\infty} \Phi^{2}\left(b_{0}\right) b \mathrm{~d} b, \quad \Phi=(K / 2) \int \gamma(t) \mathrm{d} t .
$$

3 Ch. Kittel, Elementary Solid State Physics, 1962. - American Institut of Physics Hondbook 1957.

4 D. R. BAtes, Adv. in Atomic and Molecular Physics II [1966]. - W. D. Davison, J. Phys. B London 1, 139 [1968]. 
Die Spin-Spin-Wechselwirkung besteht in einem Austausch des Edelgas-Kernspins $I$ mit dem Alkali-Valenzspin $S^{1}$. Von dem allgemeinen Hamilton-Operator für die Hyperfein-Wechselwirkung führt nur der FermiKontakt-Term

$$
\frac{8}{3} \pi \delta(B) \boldsymbol{I} \cdot \boldsymbol{S}, \quad B \triangleq \text { Edelgas-Kernort }
$$

$\mathrm{zu}$ einem wesentlichen Beitrag. Das Pauli-Prinzip fordert eine antisymmetrische Wellenfunktion und ergibt Austauschglieder, die den Wirkungsquerschnitt $\sigma_{\mathrm{SS}}$ um einen Faktor $10^{3}-10^{4}$ vergrößern. Der für den Wirkungsquerschnitt $\sigma_{\text {SS }}$ wesentliche Faktor $\eta=f(\langle i / 1\rangle)$ mit den Überlappungsintegralen $\langle i \mid 1\rangle$ (Wellenfunktionen des Valenzelektrons $|1\rangle$ und der Edelgaselektronen $|i\rangle)$ fällt jedoch unter Verwendung von Wasserstoffunktionen zu klein aus, da die wirklichen Edelgasfunktionen stärker am Kernort konzentriert sind als die Wasserstoff-Näherungsfunktionen. Für Rb erhält Herman bei Verwendung von berechneten Wellenfunktionen durchweg um $50 \%$ höhere $\eta$-Werte. Es werden auch für Na $\eta$-Werte in derselben Größenordnung wie für $\mathrm{Rb}$ gewählt und das Quadrat der Na-Funktion am Edelgas-Kernort $|\Phi(B)|^{2}$ mit Hilfe einer 3s-Wasserstoffunktion angenähert berechnet. Der Desorientierungsquerschnitt $\sigma_{\text {SS }}$ auf Grund der Spin-Spin-Wechselwirkung errechnet sich dann zu

$\sigma_{\mathrm{SS}}=\frac{2}{3}\left[8 \pi g \mu_{\mathrm{K}} \mu_{\mathrm{B}}|\Phi(B)|^{2} \eta^{2} \cdot b_{0} / \hbar \bar{v}\right]^{2} I(I+1) \sigma_{\mathrm{kin}}$,

$g$ gyromagnetisches Verhältnis des Edelgaskernes, $\mu_{\mathrm{K}}$, $\mu_{\mathrm{B}}$ Kern-, Bohr-Magneton, $\hbar$ Plancksches Wirkungsquantum, $\bar{v}$ mittlere Relativgeschwindigkeit.

In beiden Berechnungen erscheint als Parameter der kinetische Radius $b_{0}$, einmal in der 6., dann in der 4. Potenz; seine Unsicherheit trägt damit wesentlich zum Gesamtfehler des Wirkungsquerschnittes bei.

Mit Hilfe der Feldumkehr-Methode von DeHMELT ${ }^{5}$ wurden die Relaxationszeiten $t(p)$ für die Edelgase mit der natürlichen Isotopenzusammensetzung $\mathrm{He}, \mathrm{Ne}, \mathrm{Kr}$, Xe ermittelt ${ }^{6}$. Das Magnetfeld von ca. 0,5 Oe wurde mit einer Frequenz von $0,5 \ldots 20 \mathrm{~Hz}$ umgepolt. Eine

5 H. G. Dehmelt, Phys. Rev. 105, 1487 [1957].

${ }^{6}$ H. SовоLl, Diplomarbeit, Gießen 1969.

7 W. Franzen, Phys. Rev. 115, 850 [1959].
HF-Entladungslampe (60 MHz) und ein Lyot-Filter lieferten das $\mathrm{D}_{1}$-Pumplicht. Die kugelförmige Resonanzzelle $(d=6 \mathrm{~cm})$ ohne Wandbelag wurde auf die optimale Temperatur von $141{ }^{\circ} \mathrm{C}$ geheizt. Ein Photovervielfacher (6217 RCA) registrierte die Transparenzsignale, die gleichstrommäßig auf einen Oszillographen gegeben wurden. Nach FRANZEN ${ }^{7}$ können die gemessenen Relaxationszeiten $t(p)$ als Funktion des Edelgasdruckes $p$ in erster Näherung einer Kurve

$$
t(p)=\left(\text { const } D_{0} / p+\text { const }^{\prime} \sigma \cdot p\right)^{-1}
$$

angepaßt werden und dadurch der Diffusionskoeffizient $D_{0}$ sowie der Wirkungsquerschnitt $\sigma$ bestimmt werden.

In der Tabelle sind die gemessenen und berechneten Desorientierungsquerschnitte $\sigma$ des Na-Grundzustandes angegeben. Die experimentellen Querschnitte stimmen etwa mit den mit Hilfe des Dunkelrelaxations-Verfahrens gewonnenen Werten von ANDERSON ${ }^{8}$ überein. $\mathrm{Zu}$ bemerken ist jedoch, daß Anderson bei den schweren Edelgasen mit einem Gemisch aus 0,73 amagat (555 Torr) He und kleinen variablen Drucken von Ar, Kr, $\mathrm{Xe}$ arbeiten mußte, während bei der vorliegenden Messung die reinen Gase verwandt werden.

Berücksichtigt man den Beitrag der Kernspin-Wechselwirkung entsprechend der Isotopenhäufigkeit $(I \neq 0)$ im natürlichen Gemisch, so erhält man Querschnitte $\sigma_{\text {theor. }}$, die mit dem Experiment verglichen werden können. Obwohl die theoretischen Werte durch die zahlreichen Näherungen mit einem relativ großen Fehler behaftet sind, geben sie die Änderung des Wirkungsquerschnittes $\sigma_{\exp }$ von $\mathrm{He}$ bis $\mathrm{Xe}$ recht gut wieder. Insbesondere wird der wachsende Anteil der Spin-AustauschWechselwirkung infolge der Isotopenzusammensetzung deutlich. Im Gegensatz zu Rb, wo die Querschnitte kleiner sind und sich auch experimentell etwa gleiche Relaxationszeiten für $\mathrm{He}^{3}$ und $\mathrm{He}^{4}$ ergeben ${ }^{9}$, läßt bei $\mathrm{Na}$ der große Unterschied von $\sigma_{S S}$ und $\sigma_{S B}$ eine erhebliche Verkürzung der Relaxationszeit für reine Edelgasisotope mit $I \neq 0$ erwarten.

${ }^{8}$ L. W. Anderson, Nuovo Cimento 32, 1151 [1964].

9 M. Aymar-Fehrenbach, Thèse $3^{\text {me }}$ cycle, Universität Paris 1967. 